\title{
Der Australienforscher aus der Mark Brandenburg. Seit über 10 Jahren wird das Andenken an Ludwig Leichhardt wachgehalten Bernd Marx, Boblitz
}

Seit über zehn Jahren wird das Andenken an Ludwig Lechhardt wachgehalten. Niemand ahnte wohl bei der Gründung der Gesellschaft Ludwig Leichhardt e.V: Trebatsch am 2. Oktober 1990, dass sich die Ortschaft an den Ufern der Spree zu einem wahren Zentrum der Pflege und Wahrung des Vermächtnisses des Australienforschers Friedrich Wilhelm Ludwig Leichhardt im Lande Brandenburg entwickeln würde. "Es ist uns gelungen, das Andenken an den in unserer Gemeinde am 23. Oktober 1813 geborenen Naturwissenschaftler bei der Bevölkerung wachzuhalten und weitere Quellen für ein noch breiteres Lebens- und Schaffensbild zu erschließen", so Herr Andre Parade, Vorsitzender der Gesellschaft bei den Feierlichkeiten, anlässlich des 10. Jahrestages der Vereinsgründung. Auf Anregung motivierter Leichhardtfreunde und zahlreiche Heimatforscher aus der Gemeinde Trebatsch und der Region wurden neue interessante Initiativen im Verlaufe der letzten zehn Jahre aus der Taufe gehoben. Besonders hinsichtlich der Vorbereitungen zum 150. Todestag des Australienreidenden im Jahre 1998 wurde in einer umfangreichen Bandbreite die Öffentlichkeit über das Leben, Wirken und geheimnisvolle Ende des Entdeckers und Forschers berichtet. Seit Januar 1997 wurden über 300 Veranstaltungen, Vorträge, Ausstellungen, Tage der offenen Tür sowie Exkursionen in Deutschland und in Australien durchgeführt. $\mathrm{Zu}$ den erfolgreichsten Sonderausstellungen und Dokumentationen zählte:

- "Australien - Sehnsucht und Schicksal Ludwig Leichhardts",

- "Friedrich August Schmalfuß - Künstler, Schwager, Freund",

- "Ludwig Leichhardt - im Spiegel der Presse",

- "Entdecker und Entdeckungen der Welt" und

- "Die Welt ist nur ein Kleeblatt" in der Gemeinde Trebatsch sowie in den Städten Potsdam, Cottbus, Lübben und Senftenberg.

Insgesamt konnten über 20.000 Interessierte sich bei den Ausstellungen und Vorträgen mit dem Lebensweg und Schicksal des preußischen Wissenschaftlers vertraut machen, darunter über 2.000 Kinder und Jugendliche. Auch Publikationen, Aufsätze und Artikel sind über Ludwig Leichhardt und die Vereinsarbeit in der Oder-Spree-Region erschienen. Mittlerweise gibt es einen engen Kontakt und umfangreichen Informationsaustausch $\mathrm{zu}$ zahlreichen Institutionen in Australien. Schwerpunkt bildet gegenwärtig die langfristige Vorbereitung des 190. Geburtstags des bedeutenden Australienforschers im Jahre 2003. Die Gesellschaft Ludwig Leichhardt e. V. Trebatsch hat es sich zur Aufgabe gemacht, in den nächsten Monaten das vorhandene Leichhardt Archiv zu erschließen und die Ergebnisse in einer Ausstellung der Öffentlichkeit zu präsentieren. Die Mitglieder werden auch in Zukunft alles unternehmen, um die Erinnerungen an den bedeutenden Sohn der Gemeinde bei der Bevölkerung wachzuhalten und bei der jungen Generation das Interesse zu wecken. 